\title{
Fronteras y horizontes que nos dan que pensar en los tiempos de la educación en derechos humanos
}

\author{
Luisa Ripa \\ Professora e investigadora de la Universidad Nacional de Quilmes, \\ Buenos Aires, Argentina \\ luisaripa@speedy.com.ar
}

\begin{abstract}
Resumen En un curioso contexto de compromiso de hablarles en mi condición de profesora de filosofía quiero hacerme cargo de que en el escenario de los tiempos de los derechos humanos y, en particular, de la educación y los derechos humanos, hoy nos dan que pensar (y qué pensar, como decía Heidegger) las propuestas acerca de las fronteras, de los horizontes y de la comunidad latinoamericana. En primer lugar propongo reflexionar sobre el sentido de lo común que se propone como enclave que nos reúne. En segundo lugar, acerca de las fronteras y horizontes y su posible significación. Para concluir con una propuesta acerca de cuál podría ser el encuadre que nos ayude a seguir creciendo en EDH a partir de este Coloquio y sus producciones.
\end{abstract}

Palabras clave: América Latina, frontera, horizonte, derechos humanos, EDH.

A gradezco a los organizadores del V Coloquio Interamericano de A Educación y Derechos Humanos el haberme distinguido con este encargo de abrir el espacio coloquial, a pesar de mi condición de mera profesora de filosofía y no de experta en educación, como lo son todos y todas ustedes. Trataré entonces de honrar este pedido con algunas reflexiones teóricas que, sin embargo, pueden importarnos: al fin y al cabo la inútil tarea de la filosofía no hace más que prolongar la terca práctica humana de pensar lo que pasa y lo que nos pasa, insistentemente.

Tal como adelantara en el resumen, los pasos que propongo exploran el sentido del lema de este encuentro: pensar, entonces, sobre el sentido de la palabra comunidad, de la palabra frontera y horizonte, sobre los derechos humanos y sobre la tarea de índole ética que todo este pensar nos estaría indicando.

Pido disculpas por mi incapacidad de hablar en portugués: trataré de hacerlo en un castellano lento y espero que la anticipación de papeles sea útil para seguir este derrotero.

\section{Lo común y las comunidades}

En primer lugar, entonces, miremos hacia lo que nos indica la expresión comunes que califica las fronteras y horizontes en el espacio concreto de nuestra América Latina.

1.1. El diccionario de la Real Academia Española ${ }^{1}$-y confío que algo semejante acontezca con la lengua portuguesa- reconoce que el adjetivo refiere a algo que se da de ordinario, que es frecuente, que no parece raro ni extraordinario. 
Pero también algo que es compartido: lo común como aquello que no es individual o privativo de una persona o grupo sino que se amplía a un número mayor de personas o de agrupaciones. Esta característica de lo común puede abarcar tanto a propiedades, territorios como a tradiciones, intereses: todo tipo de haber humano puede ser, en distintos sentidos y en diversos ámbitos, común o compartido por varios y varias.

Pero también lo común se dice en un sentido de alguna forma peyorativo, y es lo común como lo vulgar y poco distinguido, hasta admitir incluso un sentido de despreciable.Es interesante ver la progresión de significaciones que va de lo habitual hasta lo de baja condición (1. corriente, recibido y admitido de todos o de la mayor parte; 2. ordinario, vulgar, frecuente y 3. muy sabido y bajo, de inferior clase y despreciable). Esta progresión dista de ser inocente porque en la construcción lingüística alienta la idea de que lo que se comparte y no tiene relieve por ser habitual y consabido, es jerárquicamente inferior a lo que se destaca y se distingue por sus peculiaridades, en lo posible, únicas y totalmente diferenciadas de cualquiera otra u otro sujeto.

1.2. Lo cual nos lleva a un pensamiento curioso de un autor alemán que a mediados del siglo XX escribe unas pocas páginas de relativo elogio de la masificación humana (Guardini, 1958, p. 75-84). Romano Guardini describe fenomenológicamente e interpreta los signos de lo que llama "el ocaso de la edad moderna". Inicialmente desprovisto de todo juicio de valor,explora sencillamente los signos de quiebre de la gran apuesta moderna que supo levantarse diferenciándose del medioevo y de la antigüedad. En el caso de la subjetividad -pivote moderno por excelencia- advierte la pérdida de la figura del individuo responsable y capaz, origen autónomo de decisiones y emprendimientos por efecto del crecimiento de la figura del "hombre masa".

Esta palabra no indica aquí algo desprovisto de valor, sino una estructura humana que está vinculada a la técnica y a la planificación. Como aún carece de toda tradición, e incluso se ve obligada a imponerse en contra de la tradición vigente, hace su aparición, desde luego, con el más claro carácter negativo; pero en su esencia constituye una posibilidad histórica lo mismo que otras. (Guardini, 1958, p. 76)

Se trataría, entonces, de una conformación histórica de identidad antropológica que merece ser mirada con cuidado y sin apasionamiento. Por esa razón, a partir de una definición que la distingue de las multitudes antiguas ya que "la masa, en el sentido actual, es otra cosa. No constituye una pluralidad de individuos no formados [...] sino que su estructura [...] está sometida a la ley de la producción en serie, que preside el funcionamiento de las máquinas". Esta aclaración se refiere a que modernamente también existían

... los que como parte de unamuchedumbre amorfa, se distinguían de una minoría selecta; pero la existencia de aquellos ponía de manifiesto el hecho de que, allí donde esta minoría constituía la norma de valores, precisaban existir los seres atados a la cotidianeidad como fondo y terreno en el que la minoría hincaba sus raíces. Sin embargo, también los miembros de la mayoría intentaban convertirse en minoría y realizar su vida propia. (Guardini, 1958, p. 76)

Para Guardini es un hecho que seguramente responde al fenómeno de la explosión demográfica por la cual para que miles de millones de seres humanos puedan habitar, alimentarse, trasladarse, estudiar, vestirse, informarse, etc., se vuelve necesaria esta producción masiva de alimentos, transportes, viviendas, vestidos, ofertas educativas e informaciones. Como tal no es ni bueno ni malo sino necesitado de desarrollar su ideal específico, distinto del de la modernidad. Seguramente aparece con signos de opresión y manipulación, pero también con algunas señales que -para este autor- son auspiciosas: antes que nada, el tránsito del ideal de la personalidad, en el trasfondo del ideal de la personalidad distinguida y del genio, al ideal de la persona: como el y la cada cual que vale por serlo, por ser persona, antes y más allá de sus notas y características peculiares. En segundo lugar, que en algunos casos puede advertir una especial camaradería que informa de una manera nueva los vínculos y los intercambios, con complicidades peculiares. Y, por último, que esto puede dar cuenta de que se da una solidaridad en la existencia, no solamente en emprendimientos o intereses, sino en la común condición de existentes en el mundo y en el tiempo.

Por cierto, el resultado positivo o negativo de esta nueva propuesta humana no depende de alguna fortuna o, de nuevo, de decisiones magistrales o liderazgos especiales, sino de una construcción ética, del cultivo de virtudes éticas y ciudadanas específicas que sostengan el proceso hacia el logro de una nueva y fecunda manera de ser humanos y humanas entre humanos y humanas.

...La virtud básica será ante todo la seriedad en el deseo de la verdad. Tal vez se pueda ver un paso hacia ella en la objetividad que puede apreciarse en muchas cosas. Esta seriedad impulsa a enterarse de qué se persigue con todas las habladurías sobre el progreso y la explotación de la naturaleza y a cargar con la responsabilidad que la nueva situación impone. La segunda virtud será la fortaleza; una fortaleza sin aspavientos, 
espiritual y personal, que se enfrenta con el caos amenazante [...] todavía se ha de añadir un tercer elemento: el ascetismo [...] El hombre tiene que aprender a ser dueño de sí mediante el vencimiento y la abnegación, y con ello a ser dueño de su propio poder. La libertad que da este dominio orientará aquella seriedad hacia las soluciones verdaderas, en tanto que hoy vemos como se emplea en ridiculeces una gravedad casi metafísica; hará que el mero valor se convierta en fortaleza y desenmascarará los pseudoheroísmos, en virtud de los cuales se deja inmolar el hombre, fascinado por pseudoabsolutos.

De todo esto tiene que surgir, por último, un arte espiritual de gobernar, en el cual se someta al poder con el poder; que distinga entre lo justo y lo injusto, entre el fin y los medios; que sea moderado y logre un medio dentro de los esfuerzos del trabajo y de la lucha, para que el hombre pueda vivir con dignidad y alegría. Solamente esto constituirá el verdadero poder. (Guardini, 1958, p. 84)

No parece menor la decidida crítica del autor al aristocraticismo que señoreaba sobre el ideal del individuo de personalidad distinguida, necesitado de ese fondo gris de humanos faltos de interés en el que destacar las formas geniales. Los sistemas de ganadores, de inevitable estructura piramidal, son sistemas de perdedores: con la perversa ley interna de que, a más perdedores, mayor es la altura del podio del o la ganador o ganadora. La manera como hemos naturalizado esta construcción de preferencia del genio destacado por sobre todo y todos y todas los y las demás es lo que merece ser visto y escuchado al menos con una atención distinta, haciéndonos cargo de algunas contradicciones inadvertidas en nuestros discursos habituales.

Este texto nos permite reunir las entradas del diccionario pero invirtiendo la valoración implícita de lo que advertíamos como progresión de significaciones: lo que se vuelve numéricamente habitual y compartido, precisamente por su condición de no relevante ni destacable sino falto de peculiaridad y distinción es, por eso mismo, condición de nuevo alumbramiento de la subjetividad como persona: más allá de sus condiciones, más allá de sus capacidades diferenciales y aptas para destacarla, más allá, incluso, de las conductas precisas - correctas o perversas-, la condición fundamental se da por el común hecho de ser humanos, de ser humanas. Y el reconocimiento de esta condición común posibilita una nueva manera de reconocerse, de reconocer al otro y de respetarse y respetarlo como tal.
Por último, para este primer punto, una consideración sobre la comunidad.

En efecto: la condición de intereses y emprendimientos comunes nos convierte en un tipo peculiar de comunidad: la comunidad de los y las que nos venimos reuniendo para conversar, coloquiar, sobre educación y derechos humanos.

Esta categoría tiene una larga tradición no exenta de conflictos en la reflexión filosófica. Constituyó el lema de la convocatoria que en 2012 hiciéramos para las II Jornadas de Intercambio en Extensión Universitaria. $^{2}$

Al respecto, dos consideraciones: una tiene que ver con el origen de la comunidad y otro con su sentido. En el primer caso nos preguntamos $-\mathrm{y}$ la historia del pensamiento es la de su debate interminable- acerca de si la comunidad surge como una instancia segunda respecto del individuo que sería la unidad primera o si lo originario es la comunidad, el estado grupal -y hasta indiferenciado en las primeras etapas del desarrollo- de la que se separa por un proceso posterior el individuo y hasta el logro de la individualidad.

En cuando a su sentido también se nos abre una larga y clásica discusión: ¿los hombres y mujeres formamos comunidades porque no nos bastamos como individuos o porque de alguna manera esta realidad nos cumple como personas, no en términos utilitarios sino de gozo y de plenitud?

En la tradición platónica tenemos tres mitos que avalan la tesis de la necesidad de reunirse: el mito del andrógino y el del nacimiento de Eros, en el Banquete y el mito de Prometeo, en el Protágoras. El andrógino es una figura de humanidad inicial: completa, esférica y poseedora de cuatro extremidades... lo que la hacía sumamente ágil y competente... pero que fuera "seccionada" en dos - origen de los sexos- y para siempre necesitada de buscar la completud en su otra mitad: sea del mismo sexo (masculino, por cierto) o del sexo opuesto, según como haya sido su situación original. Eros, por su parte, es un dios hijo de la indigencia-Penía- y de la riqueza, de la empresa, el logro -Porós-. Por eso el amor es búsqueda indigente de lo que le falta y no posee pero es búsqueda poderosa y llena de energía durante toda la vida y en todo en la vida. Por fin, en el mito del origen de la humanidad que se relata en el Protágoras se establece que los humanos debieron reunirse para defenderse de las fieras salvajes y las inclemencias del tiempo... aunque en la ciudad se encontraron con los nuevos peligros de los enfrentamientos entre pares que producen la guerra y por eso necesitaron que Zeus les enviara las virtudes

\footnotetext{
2. Segundas Jornadas de Intercambio de Extensión Universitaria "Comunidad y Comunidades". Universidad Nacional de Quilmes, 15 y 16 de octubre de 2012. Cada proyecto de extensión de la UNQ -a los que se sumaron alguno de otra universidad del conurbano- era invitado a presentar el proyecto, sus intereses, realizaciones y problemas y a responder qué era para ese equipo de trabajo y vinculación la comunidad. El resultado del encuentro, por lo demás fecundo, se encuentra en proceso de publicación.
} 
del pudor y la justicia para curar la ciudad de los impulsos y prácticas homicidas.

Los mitos son hermosos y repetidos hasta el cansancio por expertos y legos que hacen de esos relatos platónicos un ideal de encuentro y cooperación sin advertir que encierran una tesis a la que, por lo menos, hay que mirar con cuidado: la de que si no tuviéramos necesidad, si fuéramos hijos perfectos de dioses totales, si contáramos con capacidades individuales suficientes, si nos bastáramos cada uno y cada una para defendernos... no formaríamos comunidad. La comunidad -incluidas las parejas, los hijos- formada por necesidades, por condición de menesterosos y menesterosas encierra esa -a mi juicio terrible- afirmación de que si fuéramos más como individuos no nos reuniríamos.

En sentido contrario durante toda la edad media se desarrolló una teoría de la comunidad que insistía tanto en que en el origen como en su valor la comunidad es perfección y valiosidad intrínseca y superior. Con la categoría de la palabra griega ágape se hacía referencia a un amor de pura gratuidad y entrega. Basada especialmente en los mitos judeo cristianos suponían la tesis de que la divinidad, carente de toda necesidad, crea humanos y humanas para compartir las delicias de la creación. Y, con el dogma cristiano de la trinidad- la divinidad misma es desde el inicio comunidad y comunidad amorosa. Precisamente el nombre de amor de ágape, de banquete alude al convite a compartir la fiesta de la vida y del amor. Escuchemos un hermoso texto de Dostoievski en Los Hermanos Karamazof, cuando Aliosha sueña, un rato antes de experimentar su éxtasis con el stárets que le habla de Dios:

Por amor se ha hecho semejante a nosotros y se divierte con nosotros. Convierte el agua en vino para que no cese la alegría entre los invitados. Espera a otros; los llama continuamente desde hace siglos... Mira, ya traen vino nuevo; ahí están las vasijas.

A mi manera de ver esta teoría ha gozado de una fuerte apuesta pero una débil consolidación. En efecto: la defensa tantas veces ardiente de este amor de pura gratuidad y donación de superabundancia no evita las espiritualidades y prácticas que hacen de la menesterosidad y de la conveniencia -por ejemplo, la conveniencia de la salvación eterna- el motivo y el motor de los encuentros y abrazos.

En todo caso queda planteada la disyuntiva que intentaremos resolver en nuestro $3^{\circ}$ punto.

\section{La frontera y el horizonte: sus acepciones}

El segundo derrotero que emprenderemos está sugerido por las palabras "fronteras" y "horizontes" que parecen ser las definitorias en el espacio de la América común. ¿Qué se nos pide pensar y compartir en torno a fronteras y horizontes? Partiremos del primer vocablo para centrarnos en el de horizonte, lleno de sugerencias en la filosofía actual.

\subsection{Frontera y límite}

En primer lugar, la frontera, lo que está enfrente, tiene la significación de límite. Tomado en sentido geográfico como el "borde" de los países se constituye en la línea que limita y distingue a un estado de otro. Pero trascendiendo metafóricamente el escenario de las geografías concretas, las fronteras son figura de todo lo que se levanta como un muro infranqueable, algo más allá de lo cual no se puede avanzar. No sólo un pueblo o un gobierno que no pueden trasgredir ese paso sino también como definiciones de vida, ideológicas, éticas o culturales, las fronteras son esos límites.

No pocas veces, entonces, la frontera se tiñe de colores seductores y míticos: constituyéndose en una especie de espacio total "llegar a la frontera" es, de alguna manera, colmar el derrotero posible. Y superar las fronteras es abrirse a espacios no previstos ni disponibles. De la imagen ingenua de exploradores y conquistadores que derriban fronteras habituales para abrir mundos nuevos hemos llegado hoy a concepciones tales como la de la multiplicidad de universos que nos desafían a pensar contra toda lógica y contra toda imaginación en una diversidad de totalidades cuyas fronteras son absolutamente imprecisas e indisponibles.

Las fronteras son esas líneas y esos muros donde se recorta el mundo conocido y abarcable pero que, a la vez, nos tientas con la empresa de transgredirlas y superarlas en una ubicación impredecible. Más allá de la frontera estamos en peligro, quizá, pero seguramente estamos también ante una riqueza novedosa.

La traducción más frecuente y más manejable para nosotros de la noción de frontera es, entonces, esta del "límite". Los límites, lo que limita, es lo que pone fin, pero también, y por eso mismo, lo que define. El límite de un país es el de su frontera que dice que más allá ya no existe como tal, pero, a la vez, el límite de un país es el contorno que lo dibuja y precisa, dándole la figura que le pertenece y que lo retrata singularmente. Desde la infancia aprendemos a dibujar torpemente este contorno al que identificamos como patria. Si en un sentido negativo hablamos 
de "llegar al límite de las fuerzas", por ejemplo, también decimos que "esto es mi límite" para definir la corrección de una conducta que no admite margen porque así somos, esto corresponde a nuestra integridad, es lo que nos integra y especifica.

Lo que finaliza define, y porque define, también vincula. En otro texto del autor que citáramos se refiere a la concepción del mundo como limitado o como infinito, pero también como abierto o como hermético. Precisamente la concepción de un mundo infinito, sin bordes ni límites nos arriesga a pensarlo como un mundo hermético, cerrado sobre sí, incapaz de ser trasgredido ni de llevarnos a otra orilla, otra realidad, otro mundo. Cuando lo propio es sin fin, pierde forma y peculiaridad y, sobre todo, pierde posibilidad de encuentro con algo "otro": Solamente el mundo limitado puede abrirse a otros mundos: tanto en un sentido macro como culturas complejas y densas, por ejemplo, como en un sentido micro como experiencias comunitarias, familiares o personales. La ley del límite es la de poder entrar en relación: "El límite auténtico cierra, pero, como 'tiene otro lado', abre también. El límite auténtico es como la piel: respira, siente, traspone de un lado al otro" (Guardini, 1963, p. 125). Esta hermosa imagen de la piel es la que nos puede representar mejor esta condición de la frontera/límite: no nos permite ir más allá porque nos circunscribe a ser esto y no lo otro, pero porque nos marca nuestro fin inaugura la posibilidad del encuentro. La in-finitud personal, social o nacional sería también, desde esta perspectiva, la condena a la soledad endogámica.

\subsection{Frontera y enfrentamiento (semejanza con el encuentro)}

Pero la palabra frontera esconde también otra posibilidad: la del enfrentamiento porque tiene que ver con el que está enfrente. El límite no solamente es posibilidad de encuentro sino también de litigio: el encuentro puede ser encontronazo y ponernos "en contra" del otro. La diferencia es motivo de apertura y enriquecimiento pero también de sospecha y hostilidad. Nuestras fronteras dan cuenta de esta ambigua realidad: pasaje de intercambio y beneficio mutuo también lo es de demarcaciones desconocedoras y herméticas, tantas veces incluso sangrientas.

Éste es el costo del encuentro: la posibilidad de la guerra.

Los educadores y educadoras conocemos la necesidad de «poner límites» y de enseñar a pelear lealmente por lo que se quiere... pero conocemos también los extremos a los que se puede llegar impulsados por el miedo a no ser nada ni nadie, el miedo a desaparecer, el miedo a que la presencia y la realidad del otro, de lo otro, de lo diferente, me amenace de desaparición y muerte. Lo que pudiera ser comercio interpersonal e intercultural fecundo se vuelve guerra por las demarcaciones, por las lenguas, por los ritos, por las identidades, por los reconocimientos.

El sociólogo de la religión Enzo Pace describe con todo detalle el proceso por el cual las diferencias de los que conviven se transforman de ser motivo de distinción y de broma a ser razón de guerra: mostrando cómo el territorio y el texto sagrado operan como materiales de modificación y progresivo enfrentamiento. Un novelista pone en boca del médico de Córdoba, Maimónides, la observación de que en esa ciudad andaluza convivían "los que adoraban a Dios el viernes, los que lo adoraban el sábado y los que lo adoraban el domingo»: convivencia que se pierde de la manera más cruel en especial en la empresa de las cruzadas...

En nuestras conclusiones, entonces, tendremos que hacernos cargo, también, de esta tensión que entrañan las fronteras y sus límites.

\subsection{Horizonte: dispensa y cobijo}

La palabra griega ópí̧ $\omega \nu$, se traduce, también, por límite. Referido a la línea imaginaria que divide el cielo de la tierra, el horizonte es la representación de la apertura total disponible. Oculto en la ciudad o en la cordillera, se abre, magnífico, en el océano, en el campo abierto o en la cima de la montaña. Su leve forma circular abraza el paisaje y construye por eso una de las metáforas más ricas de las que disponemos.

El horizonte limita pero al hacerlo permite que se destaquen las formas y los perfiles. De manera semejante a lo que la psicología gestáltica nos enseñara con tantas experiencias de fondo y figura, el horizonte es ese fondo tantas veces invisible gracias al cual se visualizan las distintas figuras de todo tipo. Esta noción es fundamental en la fenomenología husserliana: según ella el horizonte es la condición de posibilidad de que aparezcan realidades. Por ejemplo, nosotros nos estamos entendiendo gracias a que compartimos un horizonte de lenguaje, un horizonte de conocimiento, un horizonte de intereses por la educación y los derechos humanos, etc. etc.

El horizonte es entonces, el que dispensa la posibilidad de experiencia. Como tal es epocal, pertenece a un tiempo y a un espacio concreto. Es plural y seriado, de modo que hay horizontes diversos y más o menos abarcadores. Inadvertido en su función de permitir la experiencia puede ser visualizada en el contexto de otro horizonte más abarcador. De este modo juega haciendo visible y desapareciendo en esa operación en una tensión que lo afirma y lo invisiviliza.

Los horizontes son, en este sentido, oportunidades. Cuando en el coloquio hablamos de horizontes nos preguntamos en realidad por las posibilidades que se 
abren a este empeño sostenido de la educación y los derechos humanos.

Pero son, también, a su manera y por su forma, un cierto cobijo. El horizonte abriga y abraza. El horizonte protege y permite que los espacios sean recorribles, por la vista y por la marcha: que los territorios tengan dimensiones y que los proyectos tengan medidas. Nuestros horizontes son nuestras apuestas concretas a que lo que puede ser, sea. A que lo que deba ser, sea.

Rescataremos para concluir este punto, dos nociones de horizonte relacionadas con la experiencia y con la intersubjetividad.

\subsection{Horizonte y experiencia}

El filósofo e historiador Reinhart Kosellek ha elaborado unas categorías que profundizan ese sentido del horizonte. En lugar de reconocerlo como el espacio para cualquier experiencia posible, Kosellek establece una diferencia entre la experiencia y la expectativa.

La historia, según este modo de pensar, se construye entre el espacio de experiencia y el horizonte de expectativas. Las define como "categorías metahistóricas, es decir, antropológicas": se trata de una filosofía de la historia que precede a la investigación y exposición histórica misma.

Las experiencias habidas constituyen el presente del pasado, el pasado presente. Como pasado no existe, pero se hace presente en el acumular de la experiencia vivida, propia y ajena. Siguiendo expresamente la famosa meditación agustiniana respecto del tiempo, aplica a la historia este movimiento por el que se reconoce, a la vez, que el pasado no existe, tampoco el futuro y aún el presente, fugaz y fluente, que no existe como tal para poder asirlo... pero que son reales en la experiencia "del alma", como memoria, expectativa y atención, según Agustín.

Lo pasado se acumula como un suelo firme en el que nos plantamos como sujetos y como comunidades ante el futuro. No podríamos plantearnos planes ni proyectos sino basándonos en las experiencias que hemos tenido.

Pero tampoco el futuro existe: se hace presente futuro presene-por la expectativa: por el dibujo de proyectos y probabilidades que nos hacen esperar lo que aún no existe. Esta expectativa se distiende, se abre, en un horizonte particular que concreta los contenidos de lo que nos es dado esperar.

...ensayemos algunas definiciones a modo de oferta: la experiencia es un pasado presente, cuyos acontecimientos han sido incorporados y pueden ser recordados. En la experiencia se fusionan tanto la elaboración racional como los modos inconscientes del compor- tamiento que no deben o no debieran ya, estar presentes en el saber. Además de la propia experiencia de cada uno, trasmitida por generaciones o instituciones, siempre está contenida y conservada una experiencia ajena. En este sentido la historia se concibió desde antiguo como conocimiento de la experiencia ajena.

Algo similar se puede decir de la expectativa: está ligada a personas, siendo a la vez, impersonal, también la expectativa se efectúa en el hoy, es futuro hecho presente, apunta al todavía-no, a lo no experimentado, a lo que sólo se puede descubrir. Esperanza y temor, deseo y voluntad, la inquietud pero también el análisis racional, la visión receptiva o la curiosidad forman parte de la expectativa y la constituyen. (Koselleck,1993, p. 340)

Ciertamente tanto la experiencia como la expectativa tienen algo de inasibles y de sometidas a la pérdida: nunca parece haber tenido experiencia suficiente, o mejor dicho, suficiente almacenamiento de esas experiencias habidas por lo que el "espacio" que dibujan no es co-extensivo con la realidad de los hechos vividos y pasados sino que constituye un cierto recorte de lo que se solidifica. Tampoco la expectativa tiene existencia segura sino figuras que se distienden entre lo que designamos en términos de deseo, ilusión, proyecto, posible, probable...

Por fin, el vínculo entre las dos como suelo y apertura para hacer historia no establece ningún tipo de relación necesaria entre ambas: la creatividad y la libertad son los motores que las ponen en juego.

El pasado y el futuro no llegan a coincidir nunca, como tampoco se puede deducir totalmente una expectativa a partir de la experiencia. Una vez reunida, una experiencia es tan completa como pasados son sus motivos, mientras que la experiencia futura, la que se va a hacer, anticipada como expectativa, se descompone en una infinidad de trayectos temporales diferentes [...] Quien crea que puede deducir su expectativa totalmente a partir de su experiencia se equivoca. Si sucede algo de manera distinta a como se esperaba, queda escarmentado. Pero quien no basa su expectativa en su experiencia, también se equivoca. (Koselleck, 1993, p. 343-346)

Muestra la complejidad con que se tejen estas dos realidades y justifica el uso de esas categorías con su vínculo al espacio y al horizonte:

Tiene sentido decir la experiencia procedente del pasado es espacial, porque está reunida formando una totalidad en la que están simultáneamente presentes muchos estratos de tiempos anteriores, sin dar referencias de su antes ni de su después [...] Y viceversa, 
es más preciso servirse de la metáfora de un horizonte de expectativa que de un espacio de expectativa. Horizonte quiere decir aquella línea tras de la cual se abre en el futuro un nuevo espacio de experiencia, aunque aún no se lo pueda contemplar. La posibilidad de descubrir el futuro choca, a pesar de los pronósticos posibles, contra un límite absoluto, porque no es posible llegar a experimentarla. (Koselleck, 1993, p. 339)

Recuerda un chiste que le habrían hecho a Kruschev cuando afirmaba a sus camaradas que "se veía al comunismo en el horizonte". Sus compañeros le habrían dicho que la definición del diccionario dice que el horizonte es "una línea imaginaria que separa el cielo de la tierra y que se aleja cuando uno se acerca".

Con todas las precauciones que limitan la constitución efectiva - parcial, electiva consciente e inconscientemente- del espacio de experiencia y con todas la precauciones que nos impone el carácter de ficcional que tiene el horizonte de expectativa, ciertamente estatuyen necesariamente la posibilidad de vivir y de habitar en este mundo y esta historia (Ripa, 2008, p. 184-186).

No tengo que decir lo provechosa que esta teoría nos resulta a la hora de pensar en el oficio de educadores y educadoras, en especial preocupadas y preocupados por la relación de la educación con los derechos humanos.

\subsection{Fusión de horizontes}

Debemos a uno de los más grandes pensadores del siglo XX, Hans-Georg Gadamer, una elaboración muy precisa sobre el horizonte y los horizontes con la que concluiremos esta parte del trabajo.

Gadamer nace con el siglo XX y es considerado el mayor representante de la escuela hermenéutica moderna. Sus tesis y trabajos se condensan en una única obra, publicada cuando ya tenía 60 años, y que le fuera pedida por sus alumnos: "Verdad y Método", en dos tomos.

Lo que nos interesa en este comienzo del coloquio es su afirmación de que la existencia humana es, como tal, interpretación. Que vivimos en los contextosprevios desde los cuales y gracias a los cuales podemos ver, juzgar, apreciar, entender la realidad del mundo $\mathrm{y}$ de nosotros mismos. Esta tesis fuerte se une a otra tesis terminante del autor: que la existencia humana es, de entrada e inevitablemente dialógica: es decir, que existimos dialogando. Por fin, esta condición es la que pone, con este autor, en el centro del interés al lenguaje: a la realidad lingüística del mundo y de la existencia humana.

Lo primero que tenemos que decir de este pensador es que propone una reivindicación del prejuicio: "prejuicio no significa pues en modo alguno juicio falso, sino que está en su concepto el que pueda ser valorado positiva o negativamente" (Gadamer, 1977, p. 337). En contra de la ilustración y del positivismo, con su pretensión de no tener presupuestos, invita a reconocer que todos y todas llegamos a nuestra experiencia de mundo y a nuestro intercambio intersubjetivo con un contexto de juicios y valoraciones que es preciso reconocer y aceptar como nuestro propio horizonte, nuestro propio límite. De hecho, la idea de una "autoconstrucción absoluta de la razón" es también un juicio previo que abre a toda la empresa moderna, desde Descartes. Llega, incluso, a reclamar una reivindicación de la auténtica tradición y de una sana autoridad: la auténtica autoridad no tiene origen en "un acto de sumisión y de abdicación de la razón" sino que se funda

...en un acto de reconocimiento y de conocimiento: se reconoce que el otro está por encima de uno en juicio y perspectiva y que en consecuencia su juicio es preferente o tiene primacía respecto al propio. La autoridad no se otorga sino que se adquiere, y tiene que ser adquirida si se quiere apelar a ella. Reposa sobre el reconocimiento y en consecuencia sobre una acción de la razón misma que, haciéndose cargo de sus propios límites, atribuye al otro una perspectiva más acertada. Este sentido rectamente entendido de autoridad no tiene nada que ver con una obediencia ciega de comando. (Gadamer, 1977, p. 345-347) ${ }^{3}$

Por eso será fundamental entrar en diálogo: intentar el intercambio y abrir las posibilidades de experiencia del mundo y de la subjetividad. Escuchemos un largo texto pero muy claro al respecto:

Lo que caracteriza a la conversación frente a la forma endurecida de las proposiciones que buscan su fijación escrita es precisamente que el lenguaje realiza aquí en preguntas y respuestas, en el dar y el tomar, en el argumentar en paralelo y en el ponerse de acuerdo aquella comunicación de sentido [...] el fenómeno hermenéutico encierra en sí el carácter original de la conversación y la estructura de pregunta y respuesta. (Gadamer, 1977, p. 446-447)

Como nuestra percepción sensible del mundo es ineludiblemente privada, también lo son nuestros im-

\footnotetext{
3. Ambas tesis son cruciales para los problemas que enfrentamos: sin una reivindicación de la tradición no podremos reconocer la pertinencia de los saberes y las opciones de comunidades originarias, contra las cuales se desató el más feroz iluminismo, en especial en el modo del "higienismo positivista" (Vera del Barco).
} 
pulsos e intereses, y la razón que es común a todos y capaz de detectar eso que es común, se muestra impotente ante las ofuscaciones que en nosotros alimenta nuestra individualidad y una piedra de toque del posible acuerdo al que la razón nos invita. [...] La conversación deja siempre una huella en nosotros. Lo que hace que algo sea una conversación no es el hecho de habernos enseñado algo nuevo, sino que hayamos encontrado en el otro algo que no habíamos encontrado aún en nuestra experiencia del mundo. [...] La conversación posee una fuerza transformadora. Cuando una conversación se logra, nos queda algo, y algo queda en nosotros que nos transforma. Por eso la conversación ofrece una afinidad peculiar con la amistad. Sólo en la conversación (y en la risa común, que es como un consenso desbordante sin palabras) pueden encontrarse los amigos y crear ese género de comunidad en la que cada cual es él mismo para el otro porque ambos encuentran al otro y se encuentran a sí mismos en el otro. (Gadamer, 2002, p. 206-207)

Pero no hay que entender esta conversación como alguna forma de cierre o clausura de temas: su tesis es la de que la pregunta tiene prioridad respecto de las respuestas: el diálogo entonces es una forma de no descansar de preguntar y -como quería Heidegger- no preguntar retóricamente porque se supone la respuesta, sino preguntar de veras: haciéndose auténtica cuestión y poniéndonos en el riesgo de lo que pueda ser la, mejor dicho, las respuestas...

El primado de la pregunta frente al enunciado significa para la hermenéutica que cada pregunta que se comprende vuelve a preguntar a su vez.(II 61)

El fruto de la conversación, entonces, no será solamente el del reconocimiento de la relativa autoridad sino la apertura al reconocimiento de la precariedad y provisionalidad de las respuestas.

Esta manera de pensar le permite reelaborar el concepto de experiencia -tras recorrer las nociones que se dieron en la filosofía...- para concluir acerca de qué es alguien que tiene experiencia, que ha logrado experiencia:

...el hombre experimentado es siempre el más radicalmente no dogmático, que precisamente porque ha hecho tantas experiencias y ha aprendido de tanta experiencia está particularmente capacitado para volver a hacer experiencias y aprender de ellas. La dialéctica de la experiencia tiene su propia consumación no en un saber concluyente sino en esa apertura a la experiencia que es puesta en funcionamiento por la experiencia misma. (Gadamer, 1977, p. 431-432)
Una afirmación nos abre la puerta a la idea que perseguimos «la verdadera experiencia es aquella en la que el hombre se hace consciente de su finitud". Esta finitud está descrita por Gadamer en su concepto de horizonte. El horizonte tiene ese sentido fenomenológico que anticipáramos de ámbito que otorga, como fondo invisible, la percepción de las cosas y los hechos. Pero no hay «un» horizonte sino múltiples horizontes de más en más abarcativos: de este modo un horizonte detrás de un horizonte permitirá «ver» al anterior y así sucesivamente. Como si nuevos «fondos» de perspectiva convirtieran en figuras a los fondos precedentes.

El «horizonte del presente» es el resultado de tradiciones silenciosas: a partir de allí «comprender es siempre el proceso de fusión de estos presuntos horizontes» (Gadamer, 1977, p. 376-377). La clave de la interpretación estará dada, entonces, para este autor, en la posibilidad de fundir en el presente el horizonte del otro: el otro tiempo, pero, también, la otra persona. No son los contenidos o figuras concretas las que tenemos que comprender e intercambiar, sino ser capaces de llegar a comprender ese horizonte desde el cual y gracias al cual se pudieron dar tales contenidos o figuras. Tenemos aquí un horizonte anterior, no como ámbito de expectativas, como quería Koselleck, sino como posibilidad de experiencias y de encuentro. Tendremos que entrar en esta diferencia y pensarla para nuestro coloquio.

\section{Los derechos humanos como nueva era}

Ahora damos un salto para abandonar estas cuestiones tan filosóficas sobre comunidad y horizontes y entrar de lleno en el tema de los derechos humanos. En esta parte conclusiva queremos afirmar la posibilidad de que los derechos humanos, como realidad histórica y cultural de nuestro tiempo, estén inaugurando una nueva era: un modo de ser y de vivir como humanos y humanas, de definir la casa, la ciudad y la tierra de una manera novedosa y peculiar. A lo mejor estamos asistiendo al inicio de la edad de los DH. Es decir, una transformación de la cultura humana. A lo mejor... a lo mejor dentro de 100 años alguien hace historia y dice bueno "a partir del '48 se genera un movimiento y se produce esta transformación en el mundo." Las modificaciones, las representaciones sociales, el imaginario de las producciones es impresionante. Es cierto que recién empieza y que ya puede verse, creo, como otro mundo respecto del mundo moderno y posmoderno.

Propongo introducir este tema con el reconocimiento de una producción con casi tres décadas de 
vigencia, presente en muchos de los programas de estudio y exposiciones en congresos, conferencias, etc. Alrededor de los años 80 y 90 tuvo amplia difusión y simpatía la que se llamó «teoría de las generaciones» respecto de los derechos humanos. El creador fue $\mathrm{Ka}$ rel Vasek, ex Director de la División de DH y Paz de la Unesco que la presentó en el Instituto Internacional de DH en Estrasburgo en 1979. Se trata de una oferta de comprensión de los distintos tratados, de su vigencia e importancia cuya clave es la progresión histórica. De este modo explica que los primeros derechos en ser proclamados son los derechos civiles y políticos, los segundos son los derechos económicos, sociales y culturales y, por último, se anunciaba la instalación de una "tercera generación" de nuevos derechos muy particularizados en defensa de minorías.

La primera, segunda y tercera generación, además, no sólo constituiría, para este modo de entender, una realidad fácticamente dada en el acontecer histórico sino que indican una realidad de verdadero deber ser: los DCP deben ser reconocidos y vigentes antes que nada, tras ellos los DESC y, por fin, los de tercera generación. Hay quienes defienden esta teoría aún después de las críticas que sufriera y continúan hablando incluso de una cuarta generación de derechos humanos (González Álvarez y Blanco ).

Dos razones avalan esa prescripción de progreso: en primer lugar, que los DCP son de carácter negativo, es decir, ordenan a los estados que no deben intervenir limitando o dificultando la libre expresión, asociación, elección de formas de gobierno y representantes, etc. etc. Los DESC, en cambio, que establecen el derecho a la vivienda, al alimento, al trabajo, a la salud, a la educación, serían de carácter positivo porque ordenan a los estados una serie de acciones concretas que permitan a los ciudadanos y ciudadanas acceder real y dignamente a esos estándares de vida. En segundo lugar y solidariamente con la primera razón, los DCP no exigen a los estados erogación alguna, dado que lo que tienen que hacer es no hacer nada para no obstaculizar la libertad ciudadana. En cambio los DESC suponen importantes erogaciones que no siempre los estados están en condiciones de hacer y, por lo tanto, su vigencia será progresiva y de acuerdo a la disponibilidad de fondos. ${ }^{4}$

Es enorme la bibliografía de quienes aceptaron sin más esa tesis y la explican con detalle y convicción. Para completar la teoría se apeló a una relación conceptual que resultó fascinante: se la vinculó, en efecto, con la Revolución Francesa y ahora puede verse que cada "generación" cumple, a su manera, alguna de las proclamas de ese movimiento libera- dor: los DCP se vinculan con la libertad, los DESC con la igualdad y los nuevos pactos tienen que ver entrañablemente con la fraternidad. El filósofo italiano Norberto Bobbio (1991) hizo propia esta tesis y su espaldarazo consagró en muchos medios bien intencionados, inteligentes y decididamente partidarios de los DH esta manera de plantear lo se dio en llamar el "fenómeno" de los DH.

Sin embargo esta teoría de las generaciones fue justamente discutida por los sectores más comprometidos con esos derechos. Eduardo Rabossi, ${ }^{5}$ el notable estudioso -filósofo y abogado, miembro destacado de las organizaciones de DH- es entre nosotros quizá quien mejor ha mostrado la falacia oculta en esta propuesta. Aunque goza de una relativa buena salud, atribuible, de acuerdo a Rabossi (1993), a esa seducción que causa su explicitación en base a los valores de la Revolución Francesa, y su identificación con la libertad, la igualdad y -"por último"- con la fraternidad, hay razones múltiples y de peso para la defensa de lo que llamaremos la unicidad de los derechos, aunque nos encontremos en plena lucha por su instalación.

En primer lugar, la teoría de las generaciones se basa en un error histórico, en una manera particular de hacer el relato que omite la presencia de derechos sociales y económicos desde las primeras declaraciones. Sin embargo ya en la misma Declaración de la Revolución Francesa, a fines del siglo XVIII, aparece la mención de algunos de los derechos que luego se agruparon en los DESC. ${ }^{6}$ En realidad la división de los tratados se debió a una estrategia política para lograr la aprobación de las dos grandes potencias, entonces polos de la tensión mundial: Estados Unidos -más propicia a aceptar DCP pero reacia a reconocer los DESC- y la Unión Soviética -que tenía la disposición inversa-. Entonces cuando Vasek expone por primera vez la teoría de las generaciones se dirige precisamente a esos interlocutores que detentaban el máximo poder de decisión internacional y lo hace con el interés de sortear el veto posible a los documentos.

En segundo lugar y sobre todo, porque defiende una progresión en la obligación de respeto y un anclaje de los llamados DESC a la efectiva disposición de fondos. Este anclaje trabaja sobre otro error que es el de considerar que los derechos civiles y políticos por su presunto carácter de meramente negativos- no suponen gastos y los DESC, en cambio, precisan de cuantiosas sumas para ser plenamente gozados por todos y todas. Ésta es la razón profunda de la diferencia de generaciones y el recurso a los valores de la RF no hacen más que enmascarar la voluntad de relativizar

\footnotetext{
4. Ver, por ejemplo, lo que publica:http://es.wikipedia.org/wiki/Derechos_humanos.

5. Un presentación del autor está disponible enhttp://es.wikipedia.org/wiki/Eduardo_Rabossi (consultado en enero 2013).

6. Declaración de los Derechos del Hombre y del Ciudadano, adoptada por la Asamblea Nacional francesa el 26 de agosto de 1789
} 
la obligación de respeto de los DESC de modo que los países miembros pudieran firmar sin resquemores dado el resguardo de la efectiva capacidad financiera. Se trata de un error porque instalar, por ejemplo, los sistemas democráticos de leyes y garantías implica un enorme gasto para el Estado que debe invertir cuantiosas sumas para que funciones las legislaturas, los sistemas de elecciones periódicas, la difusión de derechos, etc. etc. Por otro lado, no podía revelarse de ninguna manera que se trataba de una maniobra de aceptabilidad de los distintos tratados.

La tesis opuesta, entonces, proclama la unicidad e indivisibilidad de todos los derechos: todos "valen" por igual, al mismo tiempo y no puede gozarse de derecho alguno si no se goza de todos. Respecto de la inmediatez de vigencia de los DESC Ligia Bolívarofrece una alternativa interesante a la progresión atada a la disponibilidad de fondos: dado que la comunidad de las naciones se hace corresponsable de la vigencia de todos los DH con el compromiso explícito de los estados que firman y luego ratifican esos derechos, la obligación fuerza a una solidaridad entre estados que deben responsabilizarse en común por lo que es un derecho universal. ${ }^{7}$

El minucioso señalamiento de los antecedentes de proclama de los DESC, la revelación de la causa política concreta que obligó a dividir los derechos en dos tratados, la revelación de la voluntad inconfesable de limitar la vigencia de los DESC con el argumento de falta de fondos y la refutación del carácter meramente negativo $-\mathrm{y}$ por eso no oneroso- de los primeros y el carácter positivo y muy oneroso de los segundos sostienen la proclama de la unicidad e indivisibilidad de la totalidad de los derechos

\subsection{El discurso y su sentido}

Sin embargo entendemos que sí hay un verdadero proceso, no en la legitimidad y vigencia de los derechos, sino en la apropiación efectiva de las personas y comunidades de sus contenidos y exigencias. Este proceso entiendo que puede verse como una progresión discursiva, como una ampliación en los relatos y exposiciones que los nombran y que los utilizan como clave argumentativa.

En ese sentido entiendo que podemos hablar de una progresión en la conciencia y empoderamiento subjetivo y en la ampliación objetiva de sus notas.

Debo este enfoque a dos sugerencias: la insistencia de Rabossi en considerar a los DH (también) como una cultura específica y la de Rita Segato que afirma que los DH son un "nuevo sistema de nombres" en tanto y en cuanto ponen nombre a lo que hasta ese momento sólo fueran sufrimientos, tal como es el caso de los derechos de las mujeres (Segato, 2003).

En este sentido vengo insistiendo acerca de que el discurso de los derechos humanos, en primer lugar, corre el discurso de la beneficencia. No solamente respecto del Estado, no reconocido ya como benefactor sino como obligado a cumplir con los derechos de los hombres y mujeres de la comunidad civil sino en un sentido más amplio que complica, por ejemplo, las visiones y paradigmas religiosos porque lo que corresponde por derecho no es fruto de don alguno (Ripa, 2006b). El sentido del discurso migra, entonces, de las formas de pedido o solicitud a las de exigencia y reclamo.

En segundo lugar el discurso de los DH corre el de la meritocracia. No tan evidente como el anterior lo cierto es que en principio no debemos hacer nada para merecer los DH. No dependen de ninguna especial corrección o eficacia de conducta sino que se exigen por sí mismos. Esta realidad impregna el tema de los derechos de los delincuentes -aún de los autores de delitos aberrantes y los autores de delitos de lesa humanidad- al descondicionar la vigencia de los derechos de la rectitud de los agentes. La simpatía ocasional o cercanía con algunos o algunas de estas o estos agentes puede cegar respecto de otras u otros: pero no hay límite para reivindicar que todos y todas los que hayan delinquido son sujetos de derecho, de la totalidad unitaria de los DH. Afecta, también, en forma peculiar a los sistemas educativos: en efecto, en las reglamentaciones y en el imaginario social que tiene que ver fundamentalmente con el acceso, permanencia y promoción en los decursos educativos, goza de buena salud un paradigma meritocrático que se instala como un sistema férreo de inclusión y, sobre todo, de exclusión y respecto del cual no hay una crítica seria desde la perspectiva del derecho universal al acceso a la educación. Cómo esto pueda convivir con la necesaria evaluación de los procesos y resultados de los y las estudiantes es uno de los temas centrales de nuestro tiempo.

Pero es a mi juicio la más interesante de las perspectivas la que surge de la novedad identitaria que la cultura de los derechos humanos proporciona a los y las humanas y humanos de nuestro tiempo. Para plantearla ofrezco mirar las diferencias entre el viejo "estado de derecho" que fuera durante siglos la propuesta civilizatoria de tantos filósofos y juristas y nuestros actuales "derechos humanos".

Ya algo puede intuirse en la diferencia de número gramatical: "el" derecho que debe imperar para todas y todos y "los" DH: con su peculiaridad de múltiples, diversos, históricos, pactados, heterogéneos... y uni- 
versales. Constituyen así una categoría novedosa de la que da cuenta una producción teórica impresionante en número y en diversidad de perspectivas.

Creo que podemos decir que el estado de derecho, tal como fue entendido tradicionalmente, fue pensado como un corpus normativo de carácter negativo-amenazante que opera como una especie de paraguas vigilante de que la conducta de personas y grupos no afecte los derechos e intereses de otras personas y grupos. Su vigilancia se delega en especialistas, hombres y mujeres del tema, del mundo jurídico y la salud y eficacia del estado de derecho puede medirse por la ausencia de necesidad de recurrir al aparato legal y judicial: tanto más estado de derecho cuanto menos demandas y procesos se necesiten. Por esta instalación social no imaginamos que se den cursos sobre derecho penal en villas de emergencia o talleres sobre derecho civil en un jardín de infantes.

Este último comentario nos abre a la diferencia que queremos señalar: en efecto, inundamos los espacios y las oportunidades de talleres, cursos, folletos, manuales, y toda forma de noticia y empoderamiento respecto de los derechos humanos, en general y en cuestiones específicas como son los derechos de las mujeres, de los y las discapacitadas y discapacitados, de las comunidades originarias, etc. etc.

Esto es así, a mi manera de ver, porque los $\mathrm{DH}$ como discurso pregnante, que circula de más en más entre hombres y mujeres de todas las condiciones y lugares, otorgan una nueva autoidentificación identitaria en tanto que sujeto de derecho. Los derechos humanos no son así solamente algo que tengo sino, fundamentalmente, algo que soy. La definición como sujeto de derecho es la que fundamenta la exclusión del ruego de la dádiva y del esfuerzo por merecer el derecho. Con todos los excesos y ambigüedades que este proceso implica no cabe duda, me parece, de que constituye una de las revoluciones más importantes e interesantes de la humanidad. Sus efectos apenas comienzan a vislumbrarse pero no estamos en condiciones de evaluar ni siquiera someramente los alcances de esta transformación cultural.

\subsection{La nueva semántica}

En la que fuera su última obra publicada en vida el filósofo Paul Ricoeur reflexiona sobre el reconocimiento y el desarrollo de sus tres estudios nos aportan una verdadera síntesis de su filosofía anterior. Admirado de que no haya en filosofía una teoría integral del reconocimiento -cuando hay tantas teorías del conocimiento- se propone construirla con el siguiente supuesto y derrotero: el supuesto de que la pista puede buscarse en los grandes diccionarios y el elenco de definiciones de la voces reconocer y reconocimiento, con la apuesta de que las diferencias semánticas y, sobre todo, el procedo de modificaciones de sentido aportan verdaderos filosofemas, categorías a ser pensadas filosóficamente. El derrotero que elige es el de ir de la voz verbal en sentido activo - reconocer- al sentido pasivo - ser reconocido- pasando por la forma reflexiva reconocer-se- (Ricoeur 2006, p. 11-36).En una síntesis extrema que toma lo que nos interesa para el presente trabajo, podernos decir que el recorrido nos obliga a: reconocer -la verdad y aceptarla, por ejemplo, la verdad de los límites y riesgos-, reconocerse como sujeto de derecho e imputable moralmente, también como sujeto de deseo y búsqueda de encuentro- y ser reconocido en la mutualidad del don en la que la reciprocidad no elimina la generosidad del donante sino que muestra el mayor reconocimiento posible del otro, al reconocerlo tan capaz de don como lo soy yo (Ricoeur, 2006, p. 309-326).

Lo que creo que la sugerencia ricoeuriana nos permite es defender la necesidad de conjugar los derechos de una manera nueva. Si tenemos que rechazar la historicidad de las generaciones es bueno reconocer que sí existe un proceso que ha hecho que los derechos "se dijeran", en primer lugar, en tercera persona,precisamente como ese "tertium" que media entre los y las individuos y se establece como normativa con pretensión de universalidad. También se va diciendo -si mi presunción de que el discurso de los derechos humanos aporta esa novedad de autodefinición antropológica- cada vez más en más en primera persona toda vez que los y las agentes los enuncian y los reclaman a la vez como propios y debidos.

Entiendo que justamente en este momento de explosión de la diversidad y el pluralismo con las impresionantes modificaciones de la conciencia que verifica, es el tiempo de decirlos en segunda persona.

Pero no tanto en la forma del dirigirse al otro o la otra sino sobre todo en la forma de la escucha, para la que es preciso una ética adecuada (Ripa, 2013). El mismo Ricoeur propone un "modelo" de esta empresa ética: la traducción. En efecto, tan universal como la ausencia de una lengua común es el fenómeno de la traducción que describe con categorías de acogida y hospitalidad y de decir "lo mismo, de otra manera". Escuchemos esta hermosa propuesta final

Una meditación sobre la diversidad de las lenguas, aspecto fundamental de la diversidad de las culturas, puede conducir a un interesante análisis de la manera como son resueltos prácticamente todos los problemas planteados por este fenómeno masivo de que el lenguaje no existe en ninguna parte bajo una forma universal sino solamente en una fragmentación del universo lingüístico. Sin embargo, en ausencia de toda super lengua no estamos completamente desprovistos; nos queda el recurso de la traducción que merece algo mejor que ser tratada como un fenómeno secundario, 
permitiendo la comunicación de un mensaje de una lengua en otra; bajo el título de la traducción se trata de un fenómeno universal que consiste en decir de otra manera el mismo mensaje. En la traducción el locutor de una lengua se transfiere al universo lingüístico de un texto extranjero. A la inversa, él acoge en su espacio lingüístico la palabra del otro. Este fenómeno de hospitalidad lingüística puede servir de modelo a toda comprensión en la cual la ausencia de lo que podría llamarse un tercero de sobrevigilancia pone en juego las mismas operaciones de transferir en..., y de acogen dentro de ..., de los cuales el acto de la traducción es el modelo. (Ricoeur, 2000)

\subsection{Una ética posible de la EDH}

Todo este derrotero nos lleva a plantear la posibilidad de una ética que satisfaga las demandas de esta especialidad creciente que llamamos "Educación y DH” (Ripa, 2006a). La opción por una ética se funda en la convicción de que ante el peligro de la ilusión en la esperanza, en el horizonte de expectativas y ante el riesgo de contentarse con una apuesta voluntarista, la única oferta saludable es la de plantear la ética, es decir, la corrección de la empresa a llevar adelante, responsablemente (Ripa, 2008): ni el sueño ni la mera afirmación, la tarea.

Comenzamos este largo recorrido siguiendo las sugerencias que el título del Coloquio nos hiciera: América Latina: fronteras y horizontes comunes en EDH. Nos preguntamos por el sentido de lo común, por las fronteras y los horizontes, en especial, por los horizontes confiando en que esa categoría tiene la máxima carga para la EDH.

En este lugar conclusivo voy a desarrollar tres puntos: en primer lugar, recoger algunos temas esenciales para nuestra conclusión de los distintos recorridos. En segundo lugar, un resumen de los horizontes que se abren ante nosotros y nosotras. En tercer lugar una oferta ética y su relación con la educación.

3.3.1. De los puntos recorridos propongo rescatar antes que nada, la noción de "común" que no solamente se refiere a lo habitual sino también a lo falto de distinción, de calidad de ser especial: a lo ordinario en el doble sentido de la palabra. Esta acepción que era la que a Guardini le permitía la audacia de ver allí una mejor referencia a la condición de persona sin atarla a la diversidad o riqueza peculiar de la personalidad. Unido a esto entiendo que dado que hay razones suficientes para aceptar la teoría de la comunidad por interés y necesidad o la de la comunidad por prioridad amorosa, creo que en este punto lo que puede verificarse es una opción, una elección consciente que prefiere argumentos pero confiesa ideología. Sabiendo que se trata de tal cosa propongo optar por la se- gunda teoría: adoptar la tesis de que la comunidad es prioritaria y que el "deseo de vivir juntos" (Ricoeur, 1996) está en la base de las motivaciones humanas por la felicidad y la corrección.

Hagámonos cargo, enseguida, de que estas realidades distan de ser "tranquilas" y están atravesadas no sólo por las dudas y las divergencias sino también por los conflictos y enfrentamientos. Y que estos conflictos no solamente son particulares y personales sino que afectan a la estructura misma de los valores y de los derechos humanos (Ripa, 2009 a y b). Es decir, que las fronteras vinculan pero también enfrentan, que los encuentros pueden terminar como encontronazos, como ya dijéramos.

Del rico abanico conceptual en torno a experiencia y horizonte rescatemos nociones complejizadas: que la experiencia es un espacio de alguna manera sólido donde situarnos pero que, en relación proporcional a su riqueza y profundidad nos hará más abiertos y dispuestos a novedades. Y que el horizonte nos dispensa el supuesto, el contexto desde el que entender y entendernos, pero que la diversidad de horizontes puede y debe ser trasgredida hacia una fusión que permita entender lo del otro -otro tiempo, otra persona- desde su horizonte y no sólo por sus contenidos.Que es el límite el que nos define, aunque también nos limita y en definitiva nos permite el encuentro Y, por fin, que la clave es la conversación.

En definitiva, que entre la disyuntiva de la pura multiplicidad y heterogeneidad de las lenguas y la instalación de lenguas imperiales que unifiquen el lenguaje, podemos liberar la práctica -tan universal como la ausencia de una lengua universal- de la traducción, y que ésta es un auténtico modelo ético (Ricoeur, 2000).

3.3.2. Con este bagaje, hagamos un listado de nuestros -posibles- descubrimientos que propongo como un triple horizonte:

Un horizonte antropológico: el de la identidad que se construye a partir de una base peculiar -cuyo modelo es el código genético- hacia formas del sí mismo que nos cumplan como humano capaz. Pero que lo hace gracias a la mediación de la identidad narrativa, forma de ser uno mismo a partir de los relatos de los otros y hacia los otros (Ricoeur, 1996). En este horizonte estará siempre presente la tensión entre el proceso de diferenciación y la tentación de la guerra. Pero estará siempre la oferta de ver al otro, a la otra, cabalmente como "un yo tan yo como yo" (Ripa, 2010). En este punto preciso nos es posible ver otra condición del horizonte que estuvo presente sin hacerse visible: la de la horizontalidad, la de la línea que une y hermana, contra la verticalidad que establece jerarquías y exclusiones.

Un horizonte político, del que no hablamos y que estuvo operando en silencio entre líneas: ¿qué quiere decir "América Latina"? Hablamos de un deseo que 
supera el de "vivir bien (deseo de felicidad), con y para los otros (deseo de solidaridad) y en instituciones justas ("deseo de justicia") que nos planteara Ricoeur (1996). Es el deseo de fraternidad, de solidaridad y de justicia pero concretado en la Patria Grande común que conformamos como estos países que nos entendemos en portugués y castellano, en portuñol, tantas veces. Nostramérica, creciente y desbordante. En un contexto planetario, con todas sus ambigüedades, pero con su irrupción fenomenal que le permite a Dussel afirmar que la suya es la primera "ética planetaria". Los intereses -las experiencias, las expectativas- nacionales ahora se cruzan de una manera nueva y tan poderosa que nos obliga a salir -no sólo, pero también-, de los planteos de una subjetividad y una intersubjetividad de las pequeñas comunidades. Lo que digo puede entenderse en una manera declamatoria y romántica o puede tomar en serio los conflictos de intereses y ver la manera como superarlos en una apuesta común que construya una historia nueva.

Por fin, un horizonte ético. Un horizonte en el marco de esa empresa que desde siempre ocupa y preocupa a los humanos ¿qué debo hacer? ¿Qué debo hacer, cómo debo ser para ser una buena persona?

Como soy profesora de ética me atrevo a hacerles una propuesta de síntesis. En medio de los debates entre los defensores de éticas que propongan fines o bienes a ser conseguidos y las que solamente aceptan que se puedan ofrecer principios y procedimientos justos y correctos, en medio de las discusiones sobre valores y virtudes, en medio de las propuestas complejas como las de Dussel o Ricoeur, permítanme plantear la necesidad de reconocer cuatro elementos.

Antes que nada, no es inocente el número: hay teorías que trabajan con la dualidad de elementos polares, contrarios y complementarios (por ejemplo, toda la filosofía aristotélica de acto y potencia, materia y forma, alma y cuerpo, etc.). Si tiene la ventaja de la necesaria complementridad corre siempre el peligro del dualismo jerárquico. Hay teorías, como la de Hegel o Marx, continuando tradiciones platónicas o agustinianas, que prefieren articularse con el número tres: de este modo establecer tríadas casi siempre móviles y de tránsito, toda vez que no se puede estancar en la dupla. Pero a veces se recurre al cuatro: figura de lo sólido, del mundo y de la totalidad: cuatro son los elementos antiguos, los puntos cardinales. Un cuarteto dibuja la conformación del mundo, según Heidegger y la del hombre capaz, según Ricoeur. El cuatro se planta como un edificio y así quiero presentarlo: con su techo, su piso y sus paredes.

Antes que nada, el techo: el horizonte de arriba (¿valor, ideal, fin de la vida, bien supremo?) En este lugar creo que la rotunda afirmación de Dussel es verdadera: hay algo que es un principio de contenido específico y de validez universal: la vida, la vida humana y en comunidad. Otra manera, a mi juicio, de decir lo mismo es decir "el otro, la otra": el tú humano/a. Es lo mismo porque es suya la vida y porque la vida vale por ser suya. Este espacio es un no-negociable: cualquier otra cosa podremos discutir siempre que y sólo que estemos defendiendo la vida y al otro: su dignidad, su existencia.

Pero esto se sostiene con procedimientos correctos y corre peligro si no se apoya en esas paredes sólidas: la verdad y la justicia. La búsqueda, la defensa de la vida del otro digno debe hacerse en verdad (con pretensión de verdad, como dice Dussel) y en justicia. La mentira y la injusticia vician la pretensión de respeto y de defensa del otro. Nuestra experiencia latinoamericana respecto al genocidio es iluminadora en este sentido: no hay bien personal -como la seguridad, por ejemplo- o social -como la pacificación, por ejemplo- que puedan buscarse legítimamente si no se lo hace en verdad y en justicia.

Por fin, el piso en el que se apoya este edificio ético es el tener. Superando conocidas disyuntivas entre ser y tener ("ser más o tener más") me parece que es preciso sostener que para los y las humanas y humanos ser incluye necesariamente tener. Y lo incluye porque somos corpóreos, históricos y situados en tiempo y espacio. La vida y la dignidad, en verdad y justicia pero en la forma de la apropiación: del hacer propio. Pertenencia y propiedad: lo que pertenece como propio es lo que finalmente nos constituye. En lugar de plantear disyuntivas cómodas es preciso -éticamente- criticar el tener para discutir la forma y la medida de un tener que sea justo para unos/as u otras/ os, para todos y todas (Ripa, 2005).

Con todo esto estaríamos proponiendo que la educación, en, para y con los derechos humanos tendrá que ser: una educación sensible a la diferencia. Pero, también, una educación sensible a la uniformidad y a los deseos de no diferenciarse. Una educación sensible a las tensiones y a los conflictos: de derechos, de valores, de intereses, de perspectivas... Sensible a los problemas, sin tratar de minimizarlos ni sucumbir ante ello.

Sensible al habitar concreto: a los territorios, a las lenguas y culturas del vivir posible en comunidad. Una hermosa figura de Mandrioni (1975) propone como ideal de sociedad el del "habitar creador" y figura al estado como la cerca que cuida el predio, el jardín en que se habita. Una educación que se anime a cuidar lo que otros y otras siembran, cosechan y disfrutan.

Una educación sensible al intercambio y a la cogestión del saber.

Una educación, por fin, sensible a los procesos narrativos y a las experiencias de traducción.

Si algo de esto fuera posible tenemos asegurada la morada ética para nuestros coloquios sobre metodologías y didácticas concretas. 


\section{Referências}

BLANCO ARISTÍN, Juan Ramón. Las 3 Generaciones de los Derechos Humanos. Disponível em: <http://www.tendencias21. net/derecho/Las-3-Generaciones-de-los-Derechos-Humanos_ a76.html> (consultado en enero 2013).

BOBBIO, Norberto. El tiempo de los derechos. Madrid: Sistema, 1991. 256p.

BOLÍVAR, Ligia. Derechos económicos, sociales y culturales: derribar mitos, enfrentar retos, tender puentes. Una reflexión desde la (in)experiencia de América Latina. Biblioteca Jurídica, tomo IV, UNAM, México, p. 85-101. Disponible en: < biblio.juridicas. unam.mx/libros/4/1839/7.pdf> (consultado en enero 2013). También en IIDH, <www.iidh.ed.cr/comunidades/diversidades/ docs/div_docpub $>$ (consultado en enero 2013).

DUSSEL,Enrique.Ética de la liberación en la era de la globalización y de la exclusión. Madrid:Trotta, 1998.

GADAMER, Hans-Georg. Verdad y Método I: fundamentos de una hermenéutica filosófica. Salamanca: Sígueme, 1977.

. Verdad y Método II. Salamanca: Sígueme, 2002.

GONZÁLEZ ÁlVAREZ, Roberto. Aproximaciones a los Derechos Humanos de Cuarta Generación. Publicado por la Sociedad Peruana de Ciencias Jurídicas SOPEJ. Disponible en: < http://www.sopecj. org rgaddhh.pdf $>$ (consultado en enero 2013).

GUARDINI, Romano. El ocaso de la edad moderna. Madrid: Guadarrama, 1958.

Mundo y persona. Madrid: Guadarrama, 1963.

KOSELLECK, Reinhart. Vergangene Zukunft: zur Semantik geschichtlicher Zeiten Fráncfort del Meno. Barcelona: Paidós, 1993.

MANDRIONI, Héctor. Filosofía y política. Buenos Aires: Guadalupe, 1975.

PACE, Enzo. Política y religión en el mundo moderno: el retorno de las guerras de Religión. Conferencia inaugural en las X Jornadas sobre Alternativas Religiosas. Buenos Aires, 2 de octubre de 2000. RABOSSI, Eduardo. Los derechos humanos básicos y los errores de la concepción canónica. Revista IIDH, v. 18, p. 45-73, 1993.

RICOEUR, Paul. Soi-même comme un autre. Paris: Du Seuil, 1996. Lo universal y lo histórico. Magazine littéraire, n. 390, París, septiembre de 2000.

Caminos del reconocimiento: tres estudios. México: FCE, 2006.

RIPA, Luisa. Crítica del tener: una obligación en tiempos de derechos e inequidad en desarrollo y equidad. Río IV: Ediciones ICALA, 2005. p. 548-552.

Derechos humanos y educación: triple entramado y sus ataduras. In: NAYA, L. M.; DÁVILA, P. (Coords.). El derecho a la educación en un mundo globalizado. Actas del X Congreso Internacional de Educación Comparada. Donostia, San Sebastián: Ediciones de Espacio Universitario/EREIN, Tomo II, 2006a, p. 61-71.

Des droits et des pactes entre le désir et la désillusion. Une lecture de la philosophie kantienne sous la lumière de l'étique de Paul Ricoeur. Recht und Frieden in der Philosophie Kants. Akten des X Internationalen Kant-Kongresses Herausgegeben von Valerio Rohden, Ricardo R. Terra und Guido de Almeida. Berlin-New Cork: Walter de Gruyter, 2006b.

Memoria y verdad ¿Reconciliación? In: MALLIMACI,

F. (Comp.). Modernidad, religión y memoria. Buenos Aires: Centro Franco Argentino de Altos Estudios- Colihue, 2008. p. 183-207.

. Derechos humanos, espacio de liberación. In: LIZCANO, F.; RIPA, L.; SALUM, E. (Coords.). Democracia y Derechos Humanos. México/Buenos Aires: Edición Universidad Autónoma del Estado de México/Colegio Mexiquense-Universidad Nacional de Quilmes, 2009a. p. 325-345.

Derechos en pugna: el caso de la seguridad, de la diferencia y de la excelencia académica. In: LÉRTORA, C. (Coord.). Actualidad filosófica en el Cono Sur. Ediciones FEPAI, 2009b.

. Las plurales narrativas de uno mismo y el lugar del a mí me pasa lo mismo que a usted. In: HERNÁNDEZ, Carlos Arturo (Comp.). Pluralismo. Bogotá: Ediciones Universidad Libre, 2010. p. 85-97.

El interés de la filosofía de Ricoeur en el marco de la 'explosión' de los Derechos Humanos. In: MENDOZA, Lértora, C. La agenda filosófica hoy, temas y problemas. Buenos Aires: FEPAI (en prensa), 2013.

SEGATO, Rita. La estructura fundamental de la violencia. Buenos Aires: UNQ-Prometeo, 2003

VERA DEL BARCO, Fabián. Argentina siglo XXI: de la esclavitud positivista del género al modelo de la diversidad. Conferencia en la Mesa Magistral Género y Esclavitud de Clausura delVI Coloquio Internacional Religión y Sociedad, Facultad de Filosofia y Letras. S. M. de Tucumán 27 de Junio de 2013 (inédito). 


\title{
Fronteiras e horizontes que nos permitem pensar nos tempos da educação em direitos humanos
}

\section{Resumo}

Em um curioso contexto de compromisso de falar, na minha condição de professora de filosofia, aos especialistas em educação, quero esclarecer que, no cenário dos tempos dos direitos humanos, e, em particular, da educação e os direitos humanos hoje nos dão o que pensar (e o que pensar, como dizia Heidegger) as propostas acerca das fronteiras, dos horizontes e da comunidade latino-americana. Em primeiro lugar proponho refletir sobre o sentido do comum que se apresenta como um lugar que nos une. Em segundo lugar, em relação às fronteiras e horizontes e sua possível significação. Para concluir apresento uma proposta de qual poderia ser o quadro que nos ajudaria a continuar crescendo em EDH a partir deste Colóquio e de suas produções.

Palavras-chaves: América Latina, fronteira, horizonte, direitos humanos, educação em direitos humanos.

\section{Borders and horizons that help reflect on the current state of human rights}

\begin{abstract}
Curiously, and because I am committed to talk to education experts as a philosophy teacher, I have to admit the fact that the current image of human rights, especially education and human rights, make us think (and what to think, as Heidegger said) about the borders, the horizons and the Latin-American community. First, I propose to reflect on the meaning of the common, posed as the enclave that brings us together. Second, I suggest thinking about the border and horizon and their possible connotations. Finally, I arrive at a conclusion with a suggestion about which framework, stemming from this work and its production, could help us grow in the fields of Education and Human Rights.
\end{abstract}

Key words: Latin America, frontier, horizon, human rights, human rights education.

Data de recebimento do artigo: 13/5/2013

Data de aprovação do artigo: 20/12/2013 